\title{
Short- and Long-chain Perfluoroalkyl Substances in the Water, Suspended Particulate Matter, and Surface Sediment of a Turbid River
}

\author{
Pujun Zhao ${ }^{\mathrm{a}}$, Xinghui Xia ${ }^{\mathrm{a}^{*}}$, Jianwei Dong ${ }^{\mathrm{a}}, \mathrm{Na} \mathrm{Xia}^{\mathrm{a}}$, Xiaoman Jiang ${ }^{\mathrm{a}}$, Yang $\mathrm{Li}^{\mathrm{a}}$, Yuemei Zhu ${ }^{\mathrm{b}}$ \\ ${ }^{a}$ School of Environment, Beijing Normal University, State Key Laboratory of Water \\ Environment Simulation, Beijing 100875, China \\ ${ }^{\mathrm{b}}$ School of Geography, Beijing Normal University, Beijing 100875, China
}

${ }^{*}$ Corresponding author: Xinghui Xia. School of Environment, Beijing Normal University, Beijing, 100875, China.

Tel./fax.:+86 105880 5314; email: xiaxh@bnu.edu.cn. 


\begin{abstract}
Perfluoroalkyl substances (PFASs) have attracted attentions all around the world. However, little is known about their distribution among water, suspended particulate matter (SPM), and sediment phases in rivers, especially for the short-chain PFASs. In this work, the Yellow River, the largest turbid river in the world, was selected as a case to study eleven kinds of PFASs in the three phases of rivers. These PFASs included C4-C12 perfluorinated carboxylates (PFCAs), perfluorobutyl sulfonate (PFBS), and perfluorooctansulfonate (PFOS), among which C4-C7 PFCAs and PFBS belong to short-chain PFASs, while C8-C12 PFCAs and PFOS belong to long-chain PFASs. The results showed that the total PFAS concentration ranged from $44.7 \mathrm{ng} \mathrm{L}{ }^{-1}$ to $1.52 \mu \mathrm{g} \mathrm{L}^{-1}$ in the water, from 8.19 to $17.4 \mathrm{ng} \mathrm{g}^{-1}$ in the sediment, and from 3.44 to $14.7 \mathrm{ng} \mathrm{g}^{-1}$ in the SPM. Short-chain PFASs predominated in the water and could reach up to $88.8 \%$ of the total PFAS concentration in water, while long-chain PFASs prevailed in the sediment and SPM. The PFAS concentration in SPM showed a significant negative correlation with SPM concentration in river water $(p<0.01)$. The distribution coefficients $\left(K_{\mathrm{d}}\right)$ of PFASs between sediment/SPM and water increased with their chain length and there was a positive correlation between $\log K_{d}$ and $\log K_{\text {ow }}$ (octanol-water partition coefficients). The total annual flux of all the eleven PFASs was estimated at 3.88 tons for the Yellow River into the Bohai Sea, among which the PFOA flux was the highest (0.90 tons). The widely occurrence and high concentrations of short-chain PFASs in the Yellow River indicates the shift of manufacturing focus of perfluoroalkyl chemicals from traditional long-chain ones to short-chain ones. Further studies should be conducted to evaluate the eco-environmental risks of these short-chain PFASs in water environments.
\end{abstract}

Key words: Perfluoroalkyl Substances (PFASs); Sediment; Suspended Particulate Matters (SPM); Annual Flux; Turbid River 


\section{Introduction}

Perfluoroalkyl substances (PFASs) are organofluorine compounds in which all of the hydrogen atoms on the carbon chain are replaced by fluorine atoms (Buck et al., 2011). C-F bond is the strongest covalent bond due to the strong electron drawing effect of fluorine (O'Hagan, 2008), which results in the thermally inertness of PFASs. The enduring properties and their hydrophobic/lipophobic nature make PFASs highly useful in industrial and commercial applications. Since 1950s, PFASs have been widely used as protectants for textiles, stain repellents, personal care products, pesticides, fire-fighting forms, emulsifying agents, etc. (Zushi et al., 2012).

PFASs have been detected all around the world, even in the snow from polar regions of Earth (Young et al., 2007). The concentrations of many kinds of PFASs in multiple media have been reported in recent years, including tap water (Mak et al., 2009; Schwanz et al., 2016), groundwater (Loos et al., 2010), surface water (Ju et al., 2008; Zhao et al., 2014; Lorenzo et al., 2016; Sharma et al., 2016), snow fall and biota (Yeung et al., 2009; Butt et al., 2010). Many studies found that perfluorooctanoic acid (PFOA) and perfluorooctanesulfonic acid (PFOS) were the dominant compounds and they were believed to be the final transformation products of other PFASs (Dinglasan et al., 2004; Parsons et al., 2008; Zhao et al., 2014).

Many investigators have found that PFASs could be accumulated by aquatic organisms (Conder et al., 2008; Xia et al., 2013). Previous studies have demonstrated that some PFASs may induce toxic effects on test organisms. For example, PFOA and PFOS may have reproductive and developmental toxicity on rodents (Lau et al., 2004); PFOA may exert hepatic toxic and have potential carcinogenicity on rats (Kennedy, G. L., 2004). Thus PFASs may have adverse effects on wild life and even human beings through the food web (Kannan et al., 2005). Therefore, PFOS and its salts have been added to Annex B of the Stockholm Convention on Persistent Organic Pollutants in May, 2009. The use and production of PFOS were restricted in the ratifying countries (179 parties in 2014, including China). In 2002, 3M company, the principal manufacturer (estimated $80-90 \%$ market share), phased out the production of POSF-based (perfluorooctyl sulfonyl fluoride) products (Weppner, 2000). After that, some "old" PFASs were replaced by 
"new" ones which have shorter chain length. For example, PFOS was replaced by perfluorobutane sulfonate (PFBS) by 3M Company after 2003. It has been reported that short-chain PFASs are as persistent as and more mobile in long-range transport than the long-chain homologues (Wang et al., 2015). After many years of replacing traditional PFASs, whether these substitutes have reached the same level as the old ones and they have the same environmental fate like their predecessors should be well studied.

The properties and fate of PFASs are closely related to their chain length $\left(\mathrm{C}_{n} \mathrm{~F}_{2 n+1}\right)$. Short-chain PFASs, which are defined as $\mathrm{n}<6$ for perfluorosulfonic acids (PFSAs) and $\mathrm{n}<7$ for perfluorocarboxylic acids (PFCAs) (OECD, 2011), have been detected and even prevail over long-chain PFASs in some places. For example, short-chain PFASs (C4-C6) were detected with a comparable level of the longer-chain PFASs (>C6) in the water samples from Dagu Drainage Canal (Dagu) in Tianjin, China, and perfluorobutyric acid (PFBA) was the dominant type in the short-chain analogues (Yao et al., 2014). Perfluoropentanoate (PFPnA), perfluoroheptanoate (PFHpA), and PFOA were the dominant PFASs in the Northwest Pacific Ocean and PFPnA dominated in the Bering Sea (Cai et al., 2012). However, most previous studies focused on the short-chain PFASs in water, little research is about their occurrence in suspended particulate matters (SPM) and sediments in aquatic environment. Hydrophobic organic compounds (HOCs) tend to be associated with sediment and SPM, which play important roles in controlling the fate and bioavailability of HOCs in aquatic environment, especially in turbid rivers (Ahrens et al., 2010; Dong et al., 2013). Because the fluorocarbon chains of PFASs are both hydrophobic and lipophobic, their distribution may be different from other well studied HOCs in rivers. However, little is known about the distribution of short-chain PFASs among water, SPM, and sediment.

Because the Yellow River is the largest turbid river in the world (Liu et al., 2013), this study took it as a typical case to investigate the PFAS distribution in turbid rivers, for both short-chain and long-chain ones. The studied short-chain PFASs included C4-C7 PFCAs and PFBS, and long-chain PFASs included C8-C12 PFCAs and PFOS. The main objective of this research was to study the occurrence of short-chain \& long-chain PFASs along the middle and lower reaches of the Yellow River, which is subject to intensive anthropogenic influences. The distribution behaviors 
between sediment and water, as well as between SPM and water were examined, and the annual flux of PFASs into the Bohai Sea was estimated.

\section{Materials and Methods}

\subsection{Chemicals and reagents}

PFOS (98\%) and PFOA (96\%) were purchased from AccuStandard Inc. (New Haven, USA). PFBA (99\%) and perfluoroundecanoic acid (PFUdA, 96\%) were obtained from Matrix Scientific Trade Co. (Columbia, USA). PFPnA (98\%), PFHpA (98\%), and perfluorononanoic acid (PFNA, 97\%) were supplied by Tokyo Chemical Industries (Tokyo, Japan). PFBS (98\%), perfluorohexanoic acid (PFHxA, 98\%), perfluorodecanoic acid (PFDA, 98\%), and perfluorododecanoic acid (PFDoA, 95\%) were purchased from Acros Organics (New Jersey, USA). The physicochemical parameters of the studied PFASs are listed in Table S1. The stock solution of these PFASs was prepared with 80:20 (v:v) methanol/water at a concentration of $200 \mathrm{mg} \mathrm{L}^{-1}$ for each kind of PFAS. Chromatography grade methanol was purchased from J.T. Baker of Phillipsburg (NJ, USA). $\left[1,2,3,4-{ }^{13} \mathrm{C}_{4}\right]$ perfluorooctanoic acid (mass labelled PFOA, MPFOA) $(\geq$ 99\%), used as recovery indicator, was supplied by Wellington Laboratories (Guelph, Canada). Ammonium acetate (98\%) and tetrabutylammonium hydrogen sulfate (TBA, 99\%) were obtained from Amethyst Chemicals of J\&K Scientific Ltd. (Beijing, China). Methyl-tert-butyl ether (MTBE, 99.5\%) was purchased from Acros Organics (New Jersey, USA). Any water used in this study was produced by a MilliQ purification system (Millipore, Germany).

\subsection{Sample collection}

Water and sediment samples were collected from 20 sites along the middle and lower reaches of the Yellow River, 14 of which were at the main stream and the other 6 sites were at major tributaries (Fig.1, detailed information can be seen in Table S2). The sampling process lasted from Aug. $31^{\text {st }}$ to Sept. $26^{\text {th }}$ of 2013 , which was in the normal flow period of the Yellow River. At each sampling site, three surface sediment samples $(0-10 \mathrm{~cm})$ were collected with a shovel within $1 \mathrm{~m}^{2}$ area; each sub-sample was about $200 \mathrm{~g}$ and they were stored in polyethylene (PE) valve bags, 
respectively. The samples at each sampling site were mixed thoroughly after they were transported to laboratory. Water samples were collected with a pre-rinsed metal pail from river and pre-treated on site. Specifically, each water sample was filtered through a pre-weighted glass fiber membrane $\left(\mathrm{GF} / \mathrm{F}\right.$, Whatman $\left.{ }^{\circledR}, 0.7 \mu \mathrm{m}\right)$ to separate water and SPM, and the obtained membranes were wrapped in aluminum foil. PFASs in water samples were extracted on site with solid phase extraction (SPE, details are shown in section 2.3). All the samples were stored in a cooler $\left(\right.$ at $\left.4^{\circ} \mathrm{C}\right)$ and transported to laboratory. Parameters including temperature, $\mathrm{pH}$, and conductivity of each sampling site were determined in situ. The dissolved organic carbon concentrations of water samples were measured with a Shimadzu TOC $500 \mathrm{C}$ analyzer (Japan). For the analysis of sediment organic carbon content (TOC), the samples were freeze-dried, and hydrochloric acid (1:1, $\mathrm{v} / \mathrm{v}$ ) were added to remove the inorganic carbonates, then the TOC was determined by an elemental analyzer (Vario E1, Elementar Analysensyteme GmbH, Hanau, Germany). All the results are listed in Table S1.

\subsection{Extraction of PFASs from water and sediment samples}

For the extraction of PFASs from water samples, Oasis WAX cartridges (Waters $®, 6 \mathrm{cc}, 150$ mg) were used according to a previously described method (Taniyasu et al., 2005) with some modification. Briefly, the cartridges were pre-conditioned with the following solutions in sequence:

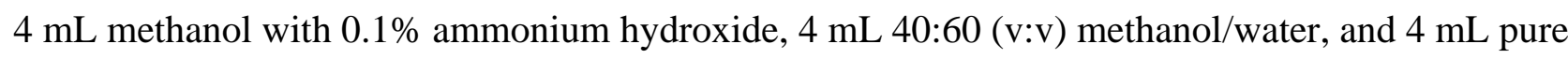
water. Then, $500 \mathrm{~mL}$ filtered water sample with recovery indicator (MPFOA, $10 \mathrm{ng}$ ) added was passed through the cartridge at the speed of 1-2 drops per second. The bottles were rinsed with pure water three times to make sure all the analytes were transferred to the cartridge. The cartridges were prevented from drying out at any time during the extraction process. After the extraction, the cartridges were transferred to laboratory and freeze-dried to remove water residual. Then the PFASs were eluted with the following solutions in sequence: $4 \mathrm{~mL}$ methanol and $4 \mathrm{~mL}$ methanol with $0.1 \%$ ammonium hydroxide. The elution was then blown to dryness under a gentle high-purity nitrogen gas flow and reconstituted with $1 \mathrm{~mL} \mathrm{80:20} \mathrm{(v:v)} \mathrm{methanol/water} \mathrm{to} \mathrm{improve}$ 
the chromatography performance (Arsenault et al., 2008). An aliquot of $300 \mu \mathrm{L}$ was transferred to a LC-MS autosampler vial with polypropylene (PP) insert tube for further instrument analysis.

Ion-pairing liquid-liquid extraction (Hansen et al., 2001) was used to extract PFASs from the sediment and SPM samples. Sediment sample of each site was well mixed and three aliquots were taken out to be freeze dried. Then they were screened with a nylon sieve (100 mesh) to remove detritus. About $5 \mathrm{~g}$ sediment was put into a PP centrifuge tube and $3 \mathrm{~mL}$ pure water was added. Then $1 \mathrm{~mL}$ of the ion-pairing agent TBA $(0.5 \mathrm{M}, \mathrm{pH}=10), 2 \mathrm{~mL}$ of $\mathrm{Na}_{2} \mathrm{CO}_{3}(0.25 \mathrm{M})$, and $5 \mathrm{~mL}$ of MTBE were added to each centrifuge tube. These tubes were shaken for $20 \mathrm{~min}$ and sonicated for $10 \mathrm{~min}$, then centrifuged to isolate the organic phase. The organic supernatant was transferred into another PP tube. This extraction process was repeated once with only MTBE added. The supernatants were combined and blown to dryness under a gentle high-purity nitrogen gas flow. Then the analytes were dissolved in $1 \mathrm{ml} \mathrm{80:20} \mathrm{(v:v)} \mathrm{methanol/water.} \mathrm{An} \mathrm{aliquot} \mathrm{of} 300 \mu \mathrm{L}$ was transferred to a LC-MS autosampler vial with polypropylene (PP) insert tube for further instrument analysis. The extraction procedure of PFASs from SPM was the same as that applied to sediment except that c.a. $1 \mathrm{~g} \mathrm{SPM}$ and $2 \mathrm{~mL}$ water were mixed. Due to the low SPM concentration in the overlying water at some sampling sites, enough SPM sample for the determination of PFASs was obtained only from ten sites. Recoveries were determined by spiking a known amount of the 11 PFASs into sediment and SPM samples and comparing the measured and theoretical values (details can be seen in SI).

\subsection{Instrumental analysis of PFASs}

PFASs were analyzed by liquid chromatography tandem mass spectrometer (LC-MS/MS). Chromatographic separation was performed on a Dionex Ultimate 3000 LC system consisting of a binary pump, an autosampler, and a column oven equipped with a 4.6×150 mm Acclaim $120 \mathrm{C} 18$ Column. The injection volume was $10 \mu \mathrm{L}$. The mobile phases were A) $5 \mathrm{mM}$ ammonium acetate solution, and B) methanol, at a flow rate of $1 \mathrm{~mL} \mathrm{~min}^{-1}$. The gradient elution started with a $0.5 \mathrm{~min}$ isocratic step at $70 \%$ of solvent $\mathrm{B}$, then ramped to $95 \%$ in $6.5 \mathrm{~min}$, held at $95 \%$ for $1 \mathrm{~min}$, and ramped down to $70 \%$ in $0.1 \mathrm{~min}$. Quantifications of PFASs were performed on a triple quadrupole 
mass spectrometer (Applied Biosystems API 3200) with electrospray negative ionization in multiple reaction monitoring (MRM) mode. Two MRM transitions were used to identify all the target compounds (except for PFBA, PFPnA, and PFHxA) while one transition was chosen for the quantification of target compounds. For PFBA, PFPnA, and PFHxA, as they can only provide one MRM transition, the confirmation was performed based one MRM transition in addition to retention time (Table S2 and Table S3). For PFOS, only linear isomer of PFOS was quantified. The instrumental parameters, e.g. declustering potential (DP), entrance potential (EP), collision energy (CE), collision cell entrance potential (CEP) and collision cell exit potential (CXP) were optimized to reach the maximum response (Table S4).

There might be some polar interferences co-eluted with the short-chain PFASs. To avoid this, the peak areas of these short-chain PFASs were calculated with the reduction of the polar interferences because their peak shape was different from the PFASs. In addition, the short-chain PFASs were not detected in some samples of the present study, suggesting that the effect of polar co-elutes was not significant.

\subsection{Quality assurance and quality control (QA/QC)}

The limit of quantification (LOQ) was defined as ten times of the background signal of solvent blank, while the limit of detection (LOD) was defined as three times of that. The LOQs for the target chemicals in the LC-MS/MS ranged from 0.0058 to $0.65 \mu \mathrm{g} \mathrm{L}^{-1}$ (Table S2). The LOQs for the target chemicals in water, sediment, and SPM samples were listed in Table S2. The correlation coefficients of the standard calibration curves were higher than 0.99 , and the repeatability of these calibration curves was confirmed prior to each set of determinations. The LC system was thoroughly cleaned with mobile phase at $90 \%$ of methanol. The blank sample of pure solvent was analyzed before each set of determination, and no target chemicals were detected. Procedural blank samples also showed no detection of the target chemicals. Recoveries of MPFOA in the water samples ranged from $81 \%$ to $124 \%$, and recoveries of the spiked PFASs in the sediment and SPM samples were between $51 \%$ and $96 \%$, which were similar to the recoveries reported by Wang et al. (2012). Each sample had three replicates and the standard deviation was less than 5\%. Each sample 
(including water, sediment, and SPM samples) had three replicates, and the PFAS concentrations in samples were not corrected according to the recoveries.

All statistical analyses were performed with SPSS 20.0 for windows (SPSS Inc., Chicago II., USA). Pearson correlation analysis was used to examine possible correlations among different variables, and the significance level was set at $p=0.05$.

\section{Results and Discussion}

\subsection{Short-chain and long-chain PFASs in the water phase}

All of the 11 studied PFASs showed very high detection frequencies in the water phase (Table 1). Only PFBS (detection frequency=85\%), PFPnA (90\%), PFHpA (95\%), and PFUdA (90\%) were not detected at some places while the rest of PFASs were detected at all the sampling sites $(n=20)$, suggesting the widely occurrence of PFASs in the Yellow River. As shown in Fig.2 (a), the total concentrations of dissolved PFASs were in the range of 44.7-263 $\mathrm{ng} \mathrm{L}^{-1}$ and 79.9-1526 $\mathrm{ng} \mathrm{L}^{-1}$ for the main stream and tributaries, respectively. Generally, tributaries had higher concentrations than the main stream $(\mathrm{T} 2>\mathrm{M} 2, \mathrm{~T} 4>\mathrm{M} 7, \mathrm{~T} 5>\mathrm{M} 8, \mathrm{~T} 6>\mathrm{M} 11)$, except that $\mathrm{T} 3 \mathrm{had}$ a lower concentration than M3. This is because the pollution source of T2 (Fenhe River) still had an effect on the main stream due to the fact that the maximum value was observed at the Fenhe River, which received many industry effluents. Besides, the site in the downstream of a dam had greater PFAS levels than those in the upstream (for example, M2>T1\&M1, M5>M4), suggesting that the dam might exert influences on the distribution of PFASs in river water. This is because the sediment re-suspension in the downstream of a dam will lead to the release of PFASs in sediment (Dong et al., 2013).

Contrast to previous studies around the world (Clara et al., 2009; Dinglasan-Panlilio et al., 2014), the biggest contribution was not PFOA (from <1\% to 24\%) among PFASs, but short-chain PFASs such as PFBA (1-45\%), PFPnA (4-58\%), and PFHxA (2-51\%) for the water phase of the Yellow River. Short-chain PFASs prevailed over long-chain PFASs in 15 sampling sites, and could reach up to $88.8 \%$ of the total PFAS concentration in the water (at M6, Xiaolangdi). The fact that some short-chain PFASs were detected at a comparable level or even a higher level than the 
longer-chain PFASs in the Yellow River is not a special case. PFBA was detected with an average of $7.59 \mathrm{ng} \mathrm{L}^{-1}$ in the Haihe River and $18.1 \mathrm{ng} \mathrm{L}^{-1}$ in Dagu Drainage Canal (China), which was dominant in both of the rivers (Yao et al., 2014). PFBS and PFBA were the predominant PFASs in the surface water of the Tangxun Lake in Wuhan, China, with relatively high average concentrations of $3660 \mathrm{ng} \mathrm{L}^{-1}$ and $4770 \mathrm{ng} \mathrm{L}^{-1}$, respectively (Zhou et al., 2013). Furthermore, statistical analysis showed that long-chain PFCAs were significantly positively correlated with PFOS $(p<0.01$ with one exception of $p<0.05)$; PFBA, PFHxA, and PFHpA were significantly positively correlated with each other $(p<0.01)$. Besides, no correlation was observed between the concentrations of short-chain PFCAs and those of long-chain PFCAs and PFOS. This interesting phenomenon may indicate that the source of short-chain PFASs is different from the long-chain PFASs. As for PFSAs, the concentration of PFOS was higher than PFBS at all the sampling sites, suggesting PFOS still prevails over PFBS in the Yellow River. The main possible sources of PFOS include food-packaging, textile, electroplating, firefighting and semiconductor industry emission sources and the precious metals and coating industry emission sources in China (Qi et al., 2016).

\subsection{Short-chain and long-chain PFASs in the SPM phase}

Long-chain PFASs and PFBS were 100\% detected in the SPM samples of the 10 studied sites, while PFBA was only detected in two of the 10 studied sites, PFPnA and PFHxA were not found for any studied sites (Table 1). The total PFAS concentration in the SPM ranged from 3.44 to 14.7 $\mathrm{ng} \mathrm{g}^{-1}$ (dry weight) as shown in Fig.2(b). This is comparable to the level reported by a previous research conducted in Tokyo Bay (Japan) that the total PFAS concentration ranged from 6.4 to $15.1 \mathrm{ng} \mathrm{g}^{-1}$ (dry weight) in the SPM fraction (Ahrens et al., 2010). Tributaries have similar PFAS levels with the main stream (T1\&M1, T3\&M3). The highest PFAS concentration was reached at the Aishan station (M11), where PFOA was the predominant compound (6.31 ng g $\mathrm{g}^{-1}$, data not shown).

Among the detected PFASs, PFOA was the predominant one with average contribution being 42\%, followed by PFUdA (20\%), PFDA (12\%), and PFDoA (12\%), while other PFASs had much lower contributions. Long-chain PFASs contributed much more than the short-chain ones. This is 
in well accordance with a previous report (Ahrens et al., 2010), which found that short-chain PFCAs were exclusively detected in water, while longer-chain PFCAs and PFSAs appeared to bind more strongly to particles. Further statistical analysis showed that several PFAS levels were significantly negatively correlated with SPM concentration $(p<0.01)$, including PFBS, PFOA, PFUdA, and PFDoA, as well as the sum of all the 11 PFASs (Fig.4). This might be due to the dilution effect of SPM and the particle concentration effect of contaminant sorption on SPM (Dong et al., 2013; Turner and Rawling, 2002). When the PFAS source or the PFAS concentration in water is stable, the increase of SPM concentration in water might decrease the PFAS concentration associated with SPM.

\subsection{Short-chain and long-chain PFASs in the surface sediment}

PFBS was only detected at 16 sites while other PFASs were detected at all the sampling sites $(\mathrm{n}=18$, Table 1). As shown in Fig.2(c), the total PFAS concentration in the sediment ranged from 8.19 to $17.4 \mathrm{ng} \mathrm{g}^{-1}$ in the main stream, and from 10.0 to $17.8 \mathrm{ng} \mathrm{g}^{-1}$ in the tributaries. For the main stream, the maximum value was observed at the Aishan station, with PFOA $\left(7.80 \mathrm{ng} \mathrm{g}^{-1}\right)$ and PFPnA (3.18 $\mathrm{n} \mathrm{g}^{-1}$ ) being the predominant compounds. For the tributaries, the highest value of the total PFAS concentration was observed at the Dongpinghu Lake with five of the eleven PFASs analyzed being the maximum among the studied tributaries. These high PFAS levels may be caused by human activities by the lake, such as some restaurants and fishery using lots of food-packaging and textile materials which are possible sources of PFASs (Qi et al., 2016). The highest PFAS value at the Dongpinghu Lake might be one reason for the highest PFAS concentration in the sediment of the Aishan station because the Dongpinghu Lake lies at the upper stream (about $30 \mathrm{~km}$ away) of the Aishan station. The total PFAS concentration in the Yellow River was at similar levels as in the Haihe River, which also empties into the Bohai Sea, with the total PFAS concentration (PFOS and C8-C12 PFCAs) in surface sediment ranged between 0.52 and $16.33 \mathrm{ng} / \mathrm{g}$ dry weight (Zhao et al., 2014). In addition, compared with other previous studies about the Yellow River (Table S5), both the short- and long- chain PFAS concentrations showed increase in the water and sediment phases, suggesting that there might be some PFAS sources. 
Long-chain PFASs contributed much more than the short-chain ones to the total PFAS concentration, with PFOA alone contributing $42.3 \%$ on average, followed by PFDA (13.9\%), PFNA (11.9\%), and PFUdA (10.3\%). Short-chain PFCAs and PFSAs presented as relatively low percentages. This may be due to the high solubility of these compounds, leading to less adsorption onto the sediment, since the solubility of PFASs decreased with increasing carbon chain length. The results obtained in this study are in good accordance with previous studies, which reported that PFOA was the highest contributor in sediment (Bao et al., 2010; Yu et al., 2013).

\subsection{Comparison of the short- and long-chain PFAS distribution among water, SPM, and sediment phases}

The distribution coefficients $\left(K_{\mathrm{d}}\right)$ of PFASs between SPM/sediment and water phases were calculated with the following equation.

$$
K_{d}=C_{s} / C_{w}
$$

where $C_{s}\left(n g \mathrm{~kg}^{-1}\right)$ is the PFAS levels in SPM or sediment, $\mathrm{C}_{\mathrm{w}}\left(\mathrm{ng} \mathrm{L}^{-1}\right)$ is the PFAS concentrations in water. In addition, the organic carbon normalized distribution coefficients $\left(K_{\mathrm{oc}}\right)$ were calculated as follows.

$$
K_{o c}=K_{d} / f_{o c}
$$

where $f_{\text {oc }}(\%)$ is the organic carbon content in SPM or sediment.

Significant variance in $K_{\mathrm{d}}$ (for both SPM and sediment-based) exists among different sampling sites, which even reached three log units (Fig.3). This is because $K_{\mathrm{d}}$ is a variable that depends on the physical, chemical, and biological conditions of the water body and varies within and among aquatic ecosystems (Zhang et al., 2013; Johansson et al., 2001). Besides, this variability may be caused by the nonequilibrium of PFASs between sediment/SPM and water in the Yellow River. The ratio of long- to short-chain PFAS concentrations in the water phase generally decreased from the upstream to the downstream (Fig.S1). This might be caused by: 1) more possible sources of short-chain PFASs existed in the downstream relative to the long-chain PFASs; 2) more and more long-chain PFASs were scavenged by the solid phases. However, the $K_{\text {oc }}$ values 
of PFASs between sediment and water obtained in this study were in good accordance with many previous researches. For example, the $\log K_{\mathrm{oc}}$ values of PFBA between sediment and water in this study were comparable to those (1.88) in a batch sorption experiment (Guelfo and Higgins, 2013). A previous research has reported that the $\log K_{\mathrm{oc}}$ was in the range of 1.70-3.80 for C5-C12 PFCAs and 1.75-2.97 for C4-C8 PFSAs (Zhao et al., 2012), which is similar to our results. These findings suggest that PFAS levels in sediment and water phase were related to each other in the Yellow River.

Lots of literature have documented the chain-length dependence of PFAS sorption (Lasier et al., 2011; Xia et al., 2013; 2015). In the present study, both the SPM-water and sediment-water distribution coefficients of PFASs increased with their chain length. Further analysis found that, for both sediment and SPM, there was a significant positive relationship between the $\log K_{\mathrm{d}}$ values of PFASs and their logarithm value of the octanol-water partition coefficients $\left(\log K_{\text {ow }}\right)$ at the main stream and the tributaries, except for the $\log K_{\mathrm{d}}$ between SPM and water in the tributaries.

Besides, when considering different functional groups, the logarithm value of the distribution coefficients $\left(\log K_{\mathrm{d}}\right)$ of PFOS $\left(\log K_{\mathrm{ow}}=4.49\right)$ was lower compared to PFNA $\left(\log K_{\mathrm{ow}}=5.48\right)$, which have the same perfluoroalkyl chain length. This founding is in contrast with other reports that the sorption of sulfonate will be stronger than the analogous carboxylic acid (Vierke et al., 2014). This is because PFOS is known to be the final transformation product of some PFASs such as (N-alkyl substituted) perfluorooctane sulfonamides and N-ethyl perfluorooctane sulfonamido ethanol (D'eon et al., 2006; Benskin et al., 2012; Zhao et al., 2016). The transformation of PFOS-precursors to PFOS happens in air, water (including waste water treatment), and soils. Therefore, PFOS in the water phase may have other sources in addition to its direct pollution sources. It has the potential to transfer from the water to solid phases, leading to the nonequilibrium between solid and water phases in the Yellow River. Thus, both the perfluoroalkyl chain length and the functional group should be considered when studying the distribution of PFASs between sediment/SPM and water. In addition, the effect of PFAS source and specific aquatic ecosystem conditions on PFAS distribution should not be ignored. 


\subsection{Estimated annual flux of PFASs to the Bohai Sea and environmental implications}

Because there are no tributaries, industrial wastewater discharges and domestic sewage flow into the Yellow River segment between the Luokou station (M12) and the river mouth, and the Luokou station is free from the seawater influence, the flux of PFASs at Luokou can represent the contribution of the river to the sea. Previous study has found that no obvious seasonal variations of PFAS levels exist between summer and winter in the Yangtze River (Pan et al., 2014). Thus, it is reasonable to assume that the annual average concentrations of PFASs are not significantly different from the results obtained in this research for the Yellow River, and the total annual mass loadings of PFASs $\left(F_{\text {total }}, \mathrm{kg}\right)$ of the Yellow River can be calculated according to the following equations.

$$
\begin{aligned}
& F_{\text {water }}=C_{\text {water }} \times Q_{\text {water }} \\
& F_{S P M}=C_{S P M} \times Q_{\text {sediment }} \\
& F_{\text {total }}=F_{\text {water }}+F_{S P M}
\end{aligned}
$$

where $F_{\text {water }}$ and $F_{\text {SPM }}$ are the annual loadings of the dissolved and the particulate-attached PFASs $(\mathrm{kg})$, respectively; $C_{\text {water }}$ and $C_{\mathrm{SPM}}$ are the annual average concentrations of dissolved and particulate-attached PFASs $\left(\mathrm{ng} \mathrm{L}^{-1}\right)$, respectively; $Q_{\text {water }}$ and $Q_{\text {sediment }}$ are the annual average water and sediment discharge $\left(\mathrm{m}^{3}, \mathrm{~kg}\right)$, respectively. Here, the PFAS levels at the Luokou station, and the annual average water discharge $\left(1.83 \times 10^{10} \mathrm{~m}^{3}\right)$ as well as the annual sediment load $\left(1.63 \times 10^{11} \mathrm{~kg}\right)$ from 2003 to 2010 (Sui et al., 2014) were used to calculate the annual flux of PFASs.

The annual flux of each kind of PFAS to the Bohai Sea is listed in Table 2. For the short-chain PFASs, dissolved form contributed much more than particulate attached form. The sum of all short-chain PFAS annual fluxes in dissolved form was $1723.6 \mathrm{~kg}$, which was 21 times higher compared to that in SPM attached form. For the long-chain PFASs, particulate attached form had bigger contribution than dissolved form. The sum of all long-chain PFAS annual fluxes in SPM attached form was $1664.1 \mathrm{~kg}$, which was three times higher compared to that in dissolved form. PFHxA ranked the first in the dissolved fraction $(851 \mathrm{~kg})$, followed by PFBA (468 kg) and PFPnA 
(300 kg). PFOA (730 kg), PFUdA (367 kg), PFDA (231 kg), and PFDoA (165 kg) were the major PFASs for the SPM attached fraction. These results suggested that the contamination of traditional PFASs still prevails. The total annual flux of the eleven PFASs was 3.88 tons, among which the PFOA flux was the highest (0.90 tons). The total annual flux of the Yellow River was much lower than the total loading of 18 kinds of PFAS in the Yangtze River to the East China Sea (20.7 tons) (Pan et al., 2014). Compared with other major rivers in the world, the PFAS flux of the Yellow River is on the same order of the Ganges River in India, where the flux of PFOS, PFOA, and PFNA discharged to the Bay of Bengal were at the level of several hundreds of kilograms per year (Yeung et al., 2009).

Although the flux of the short-chain PFASs is slightly lower than those of the traditional long-chain PFASs, the risk of these short-chain ones cannot be ignored. PFSAs with four to seven carbons may be as likely to bioaccumulate as PFOS (Lasier et al., 2011). Trace levels of PFASs were detected in soil cores from biosolids-amended soils to depths of $120 \mathrm{~cm}$, suggesting potential movement of these compounds within the soil profile over time and confirming the higher transport potential for short-chain PFASs in soils amended with municipal biosolids (Sepulvado et al., 2011). In a water-saturated sediment column test, short-chain PFCAs and PFSAs with up to six carbon atoms showed complete breakthrough while longer-chain ones were retarded, suggesting ground water may be affected by contaminated surface waters (Vierke et al., 2014). Another research also found that the occurrence of short-chain PFCAs in groundwater was consistent with the results of their column test, indicating that limited removal efficiency of short-chain PFCAs by soil (Murakami et al., 2009). Thus, when the contamination of short-chain PFASs is at the same level of long-chain PFASs in surface water, less of them will be trapped in the soil or sediment and much more will permeate into the ground water than the long-chain PFASs. This suggests that short-chain PFASs will threaten the safety of ground water supply.

\section{Conclusions}

This study mainly revealed the contamination profiles of eleven kinds of PFASs in the water, SPM, and sediment in the middle and lower reaches of the Yellow River. The results showed great 
spatial variations among different sampling sites, with total concentration ranging from $44.7 \mathrm{ng} \mathrm{\textrm {L } ^ { - 1 }}$ to $1.526 \mu \mathrm{g} \mathrm{L}^{-1}$ in water, from 3.44 to $14.7 \mathrm{ng} \mathrm{g}^{-1}$ in SPM, and from 8.19 to $17.4 \mathrm{ng} \mathrm{g}^{-1}$ in sediment. Short-chain PFASs were detected and have a considerable contribution (up to $88.8 \%$ ) in the water phase. However, they were non-detectable or at very low levels and have much lower contribution in the sediment or SPM. Several PFAS levels in SPM were significantly negatively correlated with SPM concentration $(p<0.01)$, including PFBS, PFOA, PFUdA, and PFDoA. The distribution coefficients of PFCAs between sediment and water seemed to increase with the chain length and the octanol-water partition coefficients. The results suggest that both the perfluoroalkyl chain length and the functional group should be considered when studying the distribution of PFASs between sediment/SPM and water. In addition, the effect of PFAS source and specific aquatic ecosystem conditions on PFAS distribution should not be ignored

Annual mass loading of the total eleven PFASs of the Yellow River was estimated to be 3.88 tons with traditional long-chain PFASs still prevailing. The sum of all short-chain PFAS annual fluxes in dissolved form was 21 times of that in SPM attached form, while the sum of all long-chain PFAS annual fluxes in SPM attached form was three times of that in dissolved form. Further investigations should consider the properties of SPM to explore their roles in controlling PFAS distribution, and fully evaluate the eco-environmental risks of the short-chain PFASs.

\section{Acknowledgements}

This study was supported by the National Science Foundation for Distinguished Young Scholars, (No. 51325902), the Fund for Innovative Research Group of the National Natural Science Foundation of China (Grant No. 51421065), and the National Science Foundation of China (No.51279010). 


\section{References:}

Ahrens L, Taniyasu S, Yeung LWY, Yamashita N, Lam PKS, Ebinghaus R. Distribution of polyfluoroalkyl compounds in water, suspended particulate matter and sediment from Tokyo Bay, Japan. Chemosphere 2010; 79: 266-272.

Arsenault G, Chittim B, McAlees A, McCrindle R, Riddell N, Yeo B. Some issues relating to the use of perfluoro octane sulfonate (PFOS) samples as reference standards. Chemosphere 2008; 70: 616-625.

Bao J, Liu W, Liu L, Jin YH, Ran XR, Zhang ZX. Perfluorinated compounds in urban river sediments from Guangzhou and Shanghai of China. Chemosphere 2010; 80: 123-130.

Benskin JP, Ikonomou MG, Gobas F, Woudneh MB, Cosgrove JR. Observation of a Novel PFOS-Precursor, the Perfluorooctane Sulfonamido Ethanol-Based Phosphate (SAmPAP) Diester, in Marine Sediments. Environ. Sci. Technol. 2012; 46: 6505-6514.

Buck RC, Franklin J, Berger U, Conder JM, Cousins, IT, de Voogt, P, Jensen AA, Kannan K, Mab ury SA,

van Leeuwen SP, Perfluoroalkyl and polyfluoroalkyl substances in the environment: Terminol ogy, classification, and origins. Integr. Environ. Assess. Manag. 2011; 7: 513-541

Butt CM, Berger U, Bossi R, Tomy GT. Levels and trends of poly-and perfluorinated compounds in the arctic environment. Sci. Total. Environ. 2010; 408: 2936-2965.

Cai M, Zhao Z, Yin Z, Ahrens L, Huang P, Cai M, et al. Occurrence of perfluoroalkyl compounds in surface waters from the North Pacific to the Arctic Ocean. Environ. Sci. Technol. 2012; 46: 661-8.

Clara M, Gans O, Weiss S, Sanz-Escribano D, Scharf S, Scheffknecht C. Perfluorinated alkylated substances in the aquatic environment: An Austrian case study. Water. Res. 2009; 43: 4760-4768.

Conder JM, Hoke RA, Wolf WD, Russell MH, Buck RC. Are PFCAs bioaccumulative? A critical review and comparison with regulatory criteria and persistent lipophilic compounds. Environ. Sci. Techno. 2008; 42: 995-1003.

D'eon JC, Hurley MD, Wallington TJ, Mabury SA. Atmospheric chemistry of n-methyl perfluorobutane sulfonamidoethanol, $\mathrm{C} 4 \mathrm{~F} 9 \mathrm{SO} 2 \mathrm{~N}(\mathrm{CH} 3) \mathrm{CH} 2 \mathrm{CH} 2 \mathrm{OH}$ : kinetics and mechanism of reaction with OH. Environ. Sci. Technol. 2006; 40: 1862-1868.

Dinglasan MJA, Ye Y, Edwards EA, Mabury SA. Fluorotelomer alcohol biodegradation yields poly-and perfluorinated acids. Environ. Sci. Technol. 2004; 38: 2857-2864.

Dinglasan-Panlilio MJ, Prakash SS, Baker JE. Perfluorinated compounds in the surface waters of Puget Sound, Washington and Clayoquot and Barkley Sounds, British Columbia. Mar. Pollut. Bull. 2014; 78: 173-180.

Dong J, Xia X, Zhai Y. Investigating particle concentration effects of polycyclic aromatic 
hydrocarbon (PAH) sorption on sediment considering the freely dissolved concentrations of PAHs. J. Soil Sediment 2013; 13: 1469-1477.

Hansen KJ, Clemen LA, Ellefson ME, Johnson HO. Compound-specific, quantitative characterization of organic: Fluorochemicals in biological matrices. Environ. Sci. Technol. 2001; 35: 766-770.

Johansson H, Lindstrom M, Hakanson L. On the modelling of the particulate and dissolved fractions of substances in aquatic ecosystems-sedimentological and ecological interactions. Ecol. Model. 2001; 137: 225-240.

Ju X, Jin Y, Sasaki K, Saito N. Perfluorinated surfactants in surface, subsurface water and microlayer from Dalian Coastal waters in China. Environ. Sci. Technol. 2008; 42: 3538-3542.

Kannan K, Tao L, Sinclair E, Pastva SD, Jude DJ, Giesy JP. Perfluorinated compounds in aquatic organisms at various trophic levels in a Great Lakes food chain. Arch. Environ. Con. Tox. 2005; 48: 559-566.

Kennedy GL, Butenhoff JL, Olsen GW, O’Connor JC, Seacat AM, Perkins RG, Biegel LB, Murphy SR, Farrar DG. The toxicology of perfluorooctanoate. Crit. Rev. Toxicol. 2004, 34 (4), 351-384.

Lasier PJ, Washington JW, Hassan SM, Jenkins TM. Perfluorinated chemicals in surface waters and sediments from northwest Georgia, USA, and their bioaccumulation in Lumbriculus Variegatus. Environ. Toxicol. Chem. 2011; 30: 2194-2201.

Lau C, Butenhoff JL, Rogers JM. The developmental toxicity of perfluoroalkyl acids and their derivatives. Toxicol. Appl. Pharm.2004; 198: 231-241.

Liu T, Xia XH, Liu SD, Mou XL, Qiu Y. Acceleration of denitrification in turbid rivers due to denitrification occurring on suspended sediment in oxic waters. Environ. Sci. Technol. 2013: 47(9): 4053-4061.

Loos R, Locoro G, Comero S, Contini S, Schwesig D, Werres F, et al. Pan-European survey on the occurrence of selected polar organic persistent pollutants in ground water. Water Res. 2010; 44: 4115-4126.

Lorenzo M, Campo J, Farré M, Pérez F, Picó Y, Barceló D. Perfluoroalkyl substances in the Ebro and Guadalquivir river basins (Spain). Sci. Total Environ. 2016; 540: 191-199.

Mak YL, Taniyasu S, Yeung LW, Lu G, Jin L, Yang Y, et al. Perfluorinated compounds in tap water from China and several other countries. Environ. Sci. Technol. 2009; 43: 4824-4829.

Martin MT, Mendez E, Corum DG, Judson RS, Kavlock RJ, Rotroff DM, et al. Profiling the reproductive toxicity of chemicals from multigeneration studies in the toxicity reference database. Toxicol. Sci. 2009; 110: 181-190.

Murakami M, Kuroda K, Sato N, Fukushi T, Takizawa S, Takada H. Groundwater Pollution by Perfluorinated Surfactants in Tokyo. Environ. Sci. Technol. 2009; 43: 3480-3486.

OECD,Organisation for Economic Co-operation and Development. 2011. OECD portal on perfluorinated chemicals. http:// http://www.oecd.org/ehs/pfc/ 
O'Hagan D. Understanding organofluorine chemistry. An introduction to the $\mathrm{C}-\mathrm{F}$ bond. Chem. Soc. Rev. 2008; 37: 308-319.

Pan CG, Ying GG, Zhao JL, Liu YS, Jiang YX, Zhang QQ. Spatiotemporal distribution and mass loadings of perfluoroalkyl substances in the Yangtze River of China. Sci. Total. Environ. 2014; 493: 580-587.

Parsons JR, Sáez M, Dolfing J, de Voogt P. Biodegradation of perfluorinated compounds. Reviews of Environmental Contamination and Toxicology Vol 196. Springer, 2008, pp. 53-71.

Qi YJ, Huo SL, Xi BD, Hu SB, Zhang JT, He ZS. Spatial distribution and source apportionment of PFASs in surface sediments from five lake regions, China. Scientific reports. 2016; 6.

Sharma BM, Bharat GK, Tayal S, Larssen T, Bečanová J, Karásková P, et al. Perfluoroalkyl substances (PFAS) in river and ground/drinking water of the Ganges River basin: Emissions and implications for human exposure. Environ. Pollut. 2016; 208, Part B: 704-713.

Schwanz TG, Llorca M, Farré M, Barceló D. Perfluoroalkyl substances assessment in drinking waters from Brazil, France and Spain. Sci. Total Environ. 2016; 539: 143-152.

Sepulvado JG, Blaine AC, Hundal LS, Higgins CP. Occurrence and Fate of Perfluorochemicals in Soil Following the Land Application of Municipal Biosolids. Environ. Sci. Technol. 2011; 45: 8106-8112.

Sui JJ, Yu ZG, Xu BC, Dong WH, Xia D, Jiang XY. Concentrations and fluxes of dissolved uranium in the Yellow River estuary: seasonal variation and anthropogenic (Water-Sediment Regulation Scheme) impact. J. Environ. Radioactiv. 2014; 128: 38-46.

Taniyasu S, Kannan K, So MK, Gulkowska A, Sinclair E, Okazawa T, et al. Analysis of fluorotelomer alcohols, fluorotelorner acids, and short- and long-chain perfluorinated acids in water and biota. J. Chromatogr. A 2005; 1093: 89-97.

Turner A, Rawling MC. Sorption of benzo[a]pyrene to sediment contaminated by acid mine drainage: contrasting particle concentration-dependencies in river water and seawater. Water Res. 2002; 36: 2011-2019.

Vierke L, Moeller A, Klitzke S. Transport of perfluoroalkyl acids in a water-saturated sediment column investigated under near-natural conditions. Environ. Pollut. 2014; 186: 7-13.

Wang T, Khim JS, Chen C, Naile JE, Lu Y, Kannan K, Giesy JP. Perfluorinated compounds in surface waters from Northern China: comparison to level of industrialization. Environ. Int. 2012; 42: 37-46.

Wang Z, Cousins IT, Scheringer M, Hungerbuehler K. Hazard assessment of fluorinated alternatives to long-chain perfluoroalkyl acids (PFAAs) and their precursors: Status quo, ongoing challenges and possible solutions. Environ. Int. 2015; 75: 172-179.

Weppner WA. 3M Company. 2000. Phase-out plan for POSF-based products, US Environmental Protection Agency Docket AR226-0600 2000.

Xia XH, Rabearisoa AH, Jiang XM, Dai ZN. Bioaccumulation of perfluoroalkyl substances by Daphnia magna in water with different types and concentrations of protein. Environ. Sci. 
Technol. 2013; 47: 10955-10963.

Xia XH, Rabearisoa AH, Dai ZN, Jiang XM, Zhao PJ, Wang HT. Inhibition effect of $\mathrm{Na}(+)$ and $\mathrm{Ca}(2+)$ on the bioaccumulation of perfluoroalkyl substances by Daphnia magna in the presence of protein. Environ. Toxico. Chem. 2015; 34: 429-436.

Yao YM, Zhu HH, Li B, Hu HW, Zhang T, Yamazaki E, et al. Distribution and primary source analysis of per- and poly-fluoroalkyl substances with different chain lengths in surface and groundwater in two cities, North China. Ecotox. Environ. Safe. 2014; 108: 318-328.

Yeung LWY, Yamashita N, Taniyasu S, Lam PKS, Sinha RK, Borole DV, et al. A survey of perfluorinated compounds in surface water and biota including dolphins from the Ganges River and in other waterbodies in India. Chemosphere 2009; 76: 55-62.

Young CJ, Furdui VI, Franklin J, Koerner RM, Muir DCG, Mabury SA. Perfluorinated acids in arctic snow: New evidence for atmospheric formation. Environ. Sci. Technol. 2007; 41: 3455-3461.

Yu NY, Shi W, Zhang BB, Su GY, Feng JF, Zhang XW, et al. Occurrence of Perfluoroalkyl Acids Including Perfluorooctane Sulfonate Isomers in Huai River Basin and Taihu Lake in Jiangsu Province, China. Environ. Sci. Technol. 2013; 47: 710-717.

Zhang CJ, Yan H, Li F, Hu X, Zhou Q. Sorption of short- and long-chain perfluoroalkyl surfactants on sewage sludges. J. Hazard. Mater. 2013; 260: 689-699.

Zhao SY, Ma XX, Fang SH, Zhu LY. Behaviors of N-ethyl perfluorooctane sulfonamide ethanol (N-EtFOSE) in a soil-earthworm system: Transformation and bioaccumulation. Sci. Total Environ. 2016; 554: 186-191.

Zhao XL, Xia XH, Zhang SW, Wu Q, Wang XJ. Spatial and vertical variations of perfluoroalkyl substances in sediments of the Haihe River, China. J. Environ. Sci.-China 2014; 26: 1557-1566.

Zhou Z, Liang Y, Shi Y, Xu L, Cai Y. Occurrence and Transport of Perfluoroalkyl Acids (PFAAs), Including Short-Chain PFAAs in Tangxun Lake, China. Environ. Sci. Technol. 2013; 47: 9249-9257.

Zushi Y, Hogarh JN, Masunaga S. Progress and perspective of perfluorinated compound risk assessment and management in various countries and institutes. Clean. Technol. Envir. 2012; 14: 9-20. 
Table 1 Range, median, and average concentration as well as detection frequency of PFASs in water (ng L $\left.{ }^{-1}\right)$, SPM, and sediment ( $\mathrm{ng} \mathrm{g}^{-1}$, dry wet)

\begin{tabular}{|c|c|c|c|c|c|c|c|c|c|c|c|c|}
\hline & \multicolumn{4}{|c|}{ Water $(n=20)$} & \multicolumn{4}{|c|}{$\mathbf{S P M}(\mathrm{n}=10)$} & \multicolumn{4}{|c|}{ Sediment $(n=18)$} \\
\hline & $\begin{array}{c}\text { No. of } \\
\text { detection }\end{array}$ & range & median & $\begin{array}{c}\text { average } \\
\pm \mathrm{SD}\end{array}$ & $\begin{array}{c}\text { No. of } \\
\text { detection }\end{array}$ & range & median & $\begin{array}{c}\text { average } \\
\pm \mathrm{SD}\end{array}$ & $\begin{array}{c}\text { No. of } \\
\text { detection }\end{array}$ & range & median & $\begin{array}{c}\text { average } \\
\pm \mathrm{SD}\end{array}$ \\
\hline \multicolumn{13}{|c|}{ short-chain PFASs } \\
\hline PFBS & 17 & nd-6.67 & 1.91 & $2.36 \pm 1.51$ & 10 & $0.111-0.353$ & 0.139 & $0.173 \pm 0.077$ & 16 & nd- 0.441 & 0.014 & $0.055 \pm 0.111$ \\
\hline PFBA & 20 & $6.35-53.5$ & 17.1 & $18.8 \pm 11.3$ & 2 & nd-0.532 & 0.324 & $0.324 \pm 0.208$ & 18 & $0.050-0.344$ & 0.092 & $0.124 \pm 0.079$ \\
\hline PFPnA & 18 & nd-63.9 & 26.7 & $28.9 \pm 16.0$ & 0 & & & & 18 & $0.230-2.18$ & 0.634 & $0.876 \pm 0.713$ \\
\hline PFHxA & 20 & $8.04-47.3$ & 21.8 & $22.9 \pm 13.1$ & 0 & & & & 18 & $0.013-0.190$ & 0.047 & $0.053 \pm 0.038$ \\
\hline PFHpA & 19 & nd-3.78 & 1.09 & $1.26 \pm 1.04$ & 10 & MQL-0.208 & 0.089 & $0.102 \pm 0.055$ & 18 & $0.041-0.744$ & 0.248 & $0.301 \pm 0.203$ \\
\hline \multicolumn{13}{|c|}{ long-chain PFASs } \\
\hline PFOS & 20 & $2.65-41.0$ & 7.09 & $10.8 \pm 10.6$ & 10 & $0.045-0.618$ & 0.193 & $0.252 \pm 0.178$ & 18 & $0.192-0.710$ & 0.325 & $0.341 \pm 0.112$ \\
\hline PFOA & 20 & $2.01-41.8$ & 9.11 & $11.5 \pm 9.57$ & 10 & $1.58-6.31$ & 2.16 & $2.78 \pm 1.43$ & 18 & $3.23-7.80$ & 4.66 & $4.98 \pm 1.60$ \\
\hline PFNA & 20 & $0.791-24.5$ & 2.47 & $4.83 \pm 5.36$ & 10 & MQL-0.911 & 0.383 & $0.456 \pm 0.238$ & 18 & $0.963-2.83$ & 1.32 & $1.37 \pm 0.38$ \\
\hline PFDA & 20 & $0.166-100.6$ & 1.99 & $7.50 \pm 21.5$ & 10 & $0.670-1.42$ & 0.727 & $0.787 \pm 0.295$ & 18 & $1.33-2.39$ & 1.52 & $1.57 \pm 0.24$ \\
\hline PFUdA & 18 & nd-525.5 & 1.38 & $34.7 \pm 119$ & 10 & $0.7-3.19$ & 0.98 & $1.37 \pm 0.750$ & 18 & $0.903-2.06$ & 1.13 & $1.17 \pm 0.24$ \\
\hline PFDoA & 20 & $0.149-724.6$ & 3.12 & $44.0 \pm 157$ & 10 & $0.502-1.60$ & 0.607 & $0.760 \pm 0.330$ & 18 & $0.593-1.57$ & 0.764 & $0.815 \pm 0.214$ \\
\hline
\end{tabular}
limit 
Table 2 Estimated annual flux $(F, \mathrm{~kg})$ of PFASs discharged to the Bohai Sea

\begin{tabular}{lccc}
\hline & $\boldsymbol{F}_{\text {water }}$ & $\boldsymbol{F}_{\text {SPM }}$ & $\boldsymbol{F}_{\text {total }}$ \\
\hline$\sum$ short-chain PFASs & 1723.6 & 77.9 & 1801.5 \\
\hline PFBS & 61.0 & 44.0 & 105.0 \\
PFBA & 468.1 & - & 468.1 \\
PFPnA & 300.2 & - & 300.2 \\
PFHxA & 851.4 & - & 851.4 \\
PFHpA & 43.0 & 33.9 & 76.9 \\
\hline$\sum$ long-chain PFASs & 410.8 & 1664.1 & 2074.9 \\
\hline PFOS & 129.0 & 48.6 & 177.6 \\
PFOA & 171.0 & 730.1 & 901.1 \\
PFNA & 32.4 & 121.8 & 154.2 \\
PFDA & 29.3 & 231.3 & 260.6 \\
PFUdA & - & 367.2 & 367.2 \\
PFDoA & 49.0 & 165.1 & 214.1 \\
\hline Sum. & 2134.4 & 1742.0 & 3876.4 \\
\hline
\end{tabular}




\section{List of Figure Captions}

Figure 1 Sampling sites of the middle and lower reaches of the Yellow River (M and T stands for main stream and tributary, respectively; water samples were collected at all sites, SPM samples at M1-M5, M8, M11, M12, T1, T3, sediment samples at M1, M2, M4, M5, M7-M14, T1-T6)

Figure 2 Concentration of short-chain and long-chain PFASs at different sampling sites in water (a), $\operatorname{SPM}(\mathrm{b})$, and sediment(c)

Figure 3 Relationship between the octanol-water partition coefficients (log $K_{\text {ow }}$ ) of PFASs and their sediment-water as well as SPM-water distribution coefficients $\left(\log K_{\mathrm{d}}\right)$ of the main stream and tributaries (the order of individual PFASs according to log Kow is: PFBS, PFBA, PFPnA, PFHxA, PFHpA, PFOS, PFOA, PFNA, PFDA, PFUdA, PFDoA)

Figure 4 Relationship between the PFAS concentration in SPM and SPM concentration 


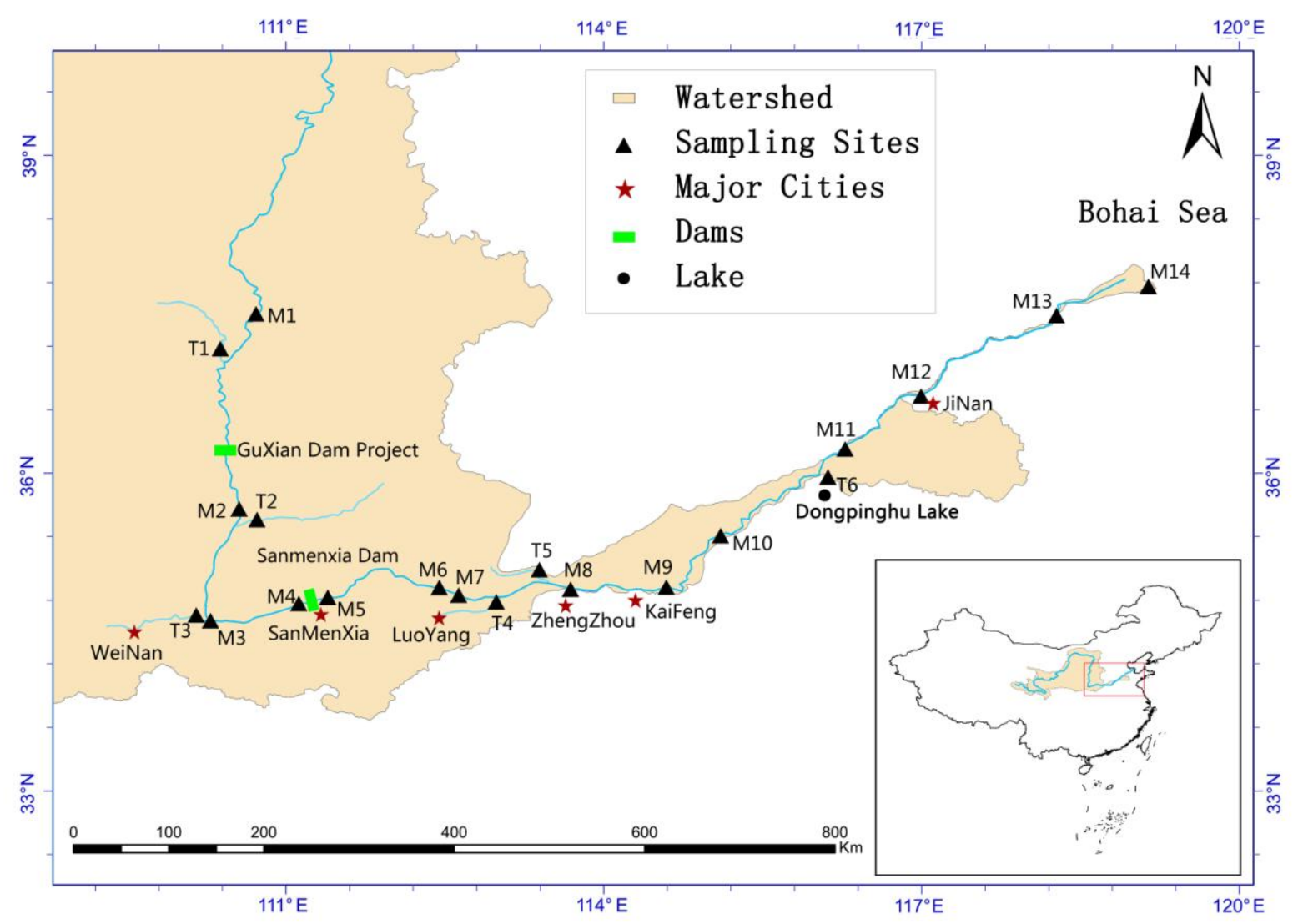

Figure 1 Sampling sites of the middle and lower reaches of the Yellow River ( $\mathrm{M}$ and $\mathrm{T}$ stands for main stream and tributary, respectively; water samples were collected at all sites, SPM samples at M1-M5, M8, M11, M12, T1, T3, sediment samples at M1, M2, M4, M5, M7-M14, T1-T6) 

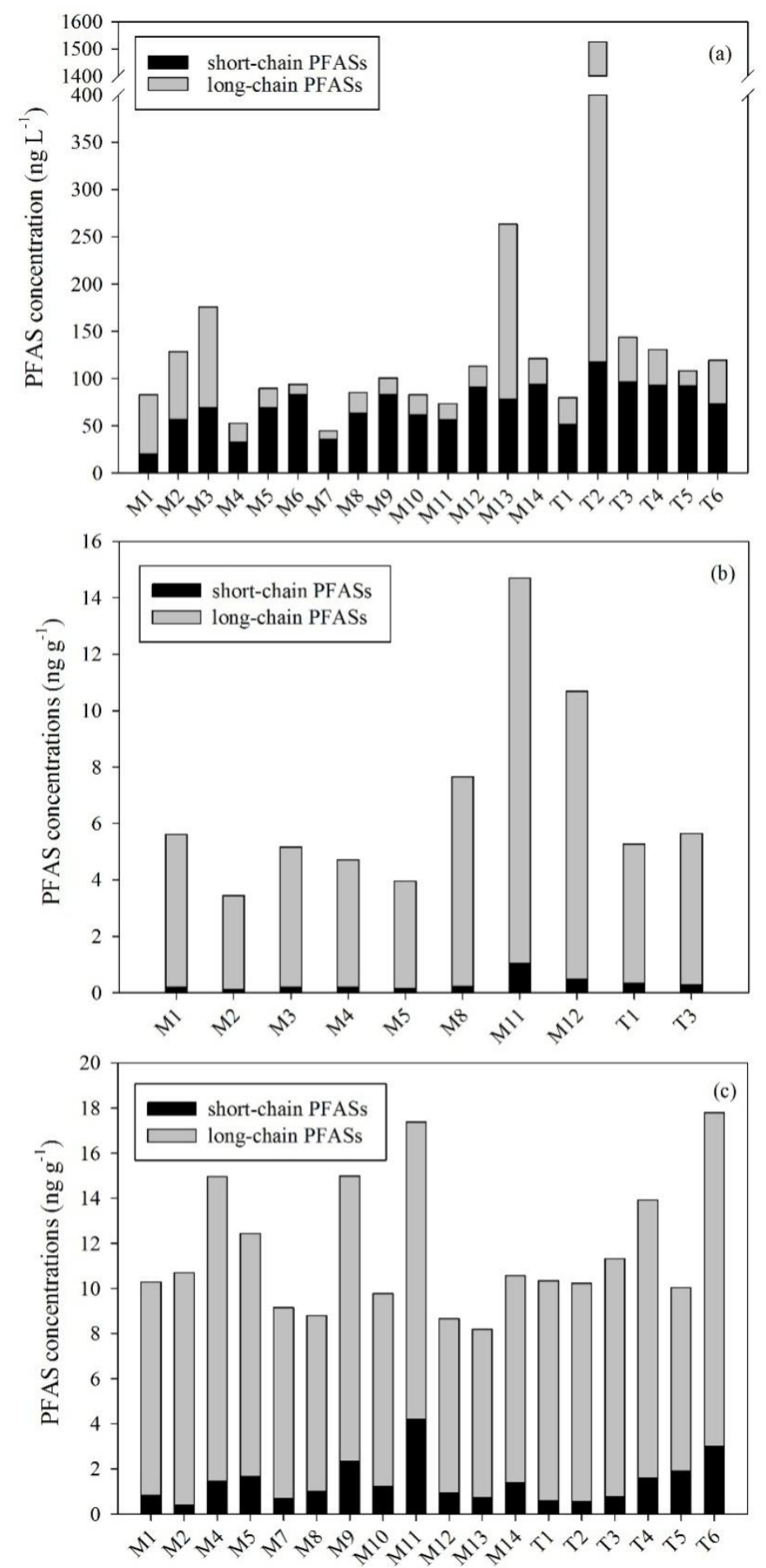

Figure 2 Concentration of short-chain and long-chain PFASs at different sampling sites in water (a), SPM (b), and sediment (c) 

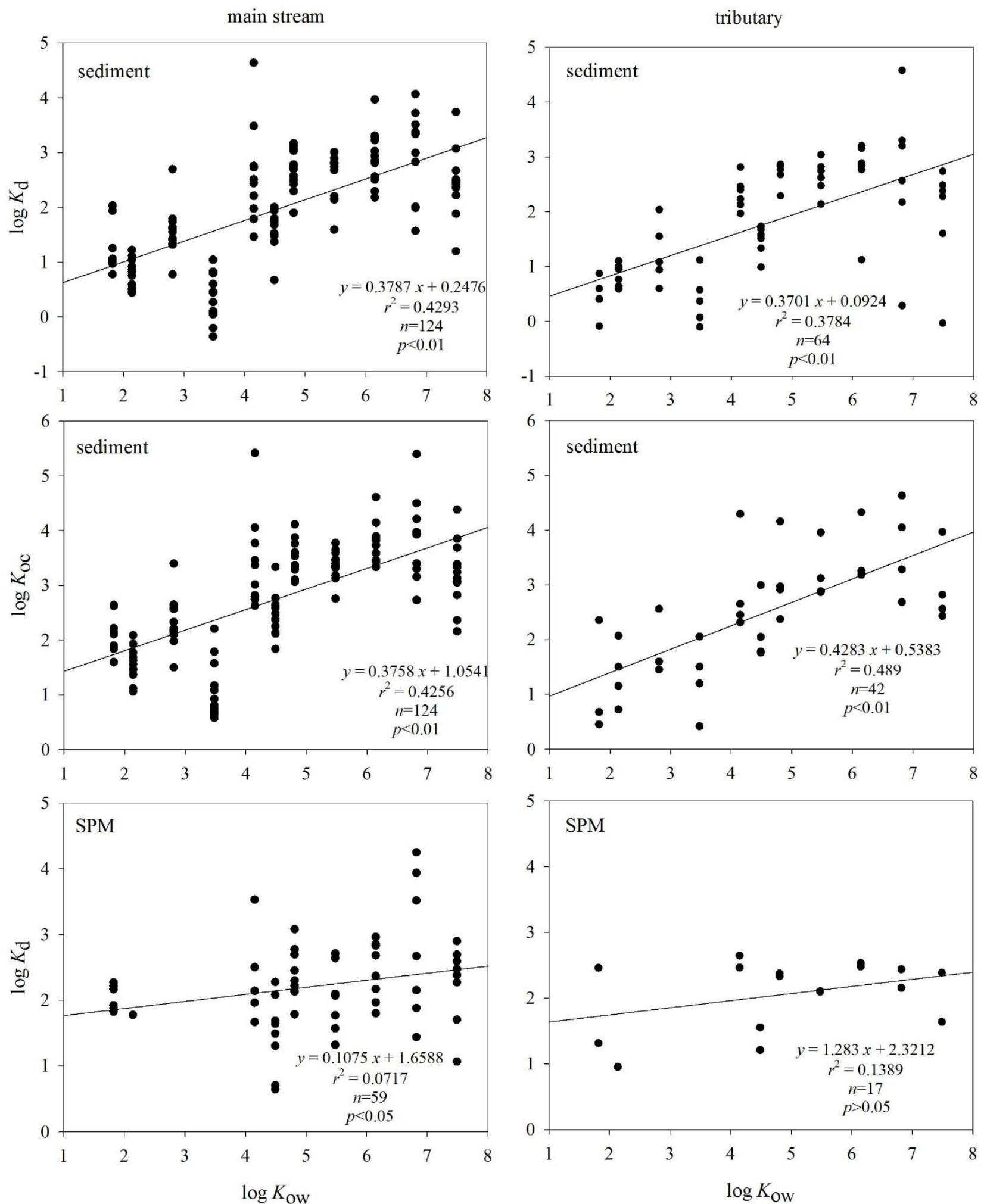

Figure 3 Relationship between the octanol-water partition coefficients ( $\left.\log K_{\text {ow }}\right)$ of PFASs and their sediment-water as well as SPM-water distribution coefficients $\left(\log K_{\mathrm{d}}\right)$ of the main stream and tributaries (the order of individual PFASs according to $\log$ Kow is: PFBS, PFBA, PFPnA, PFHxA, PFHpA, PFOS, PFOA, PFNA, PFDA, PFUdA, PFDoA) 


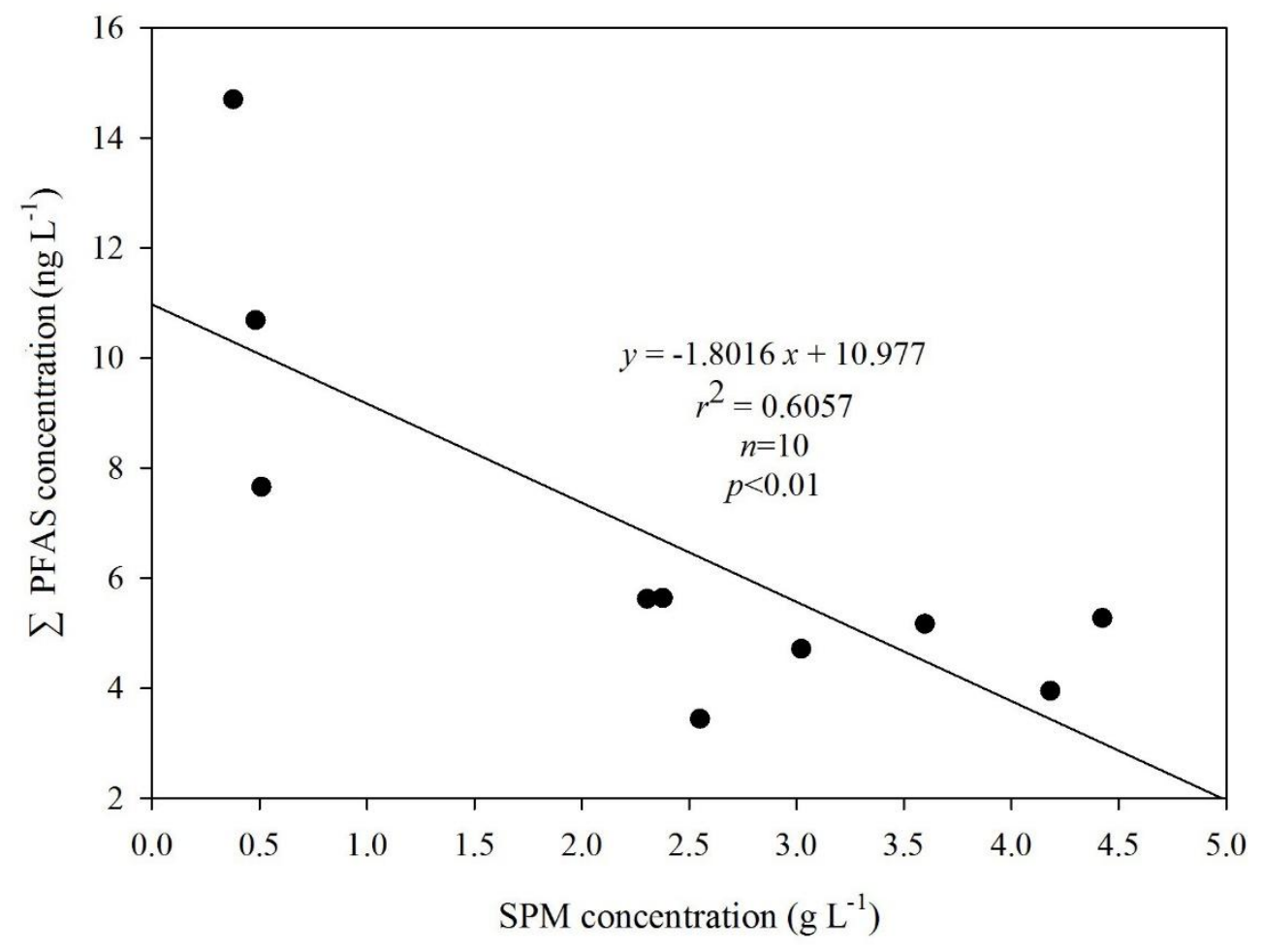

Figure 4 Relationship between the PFAS concentration in SPM and SPM concentration 


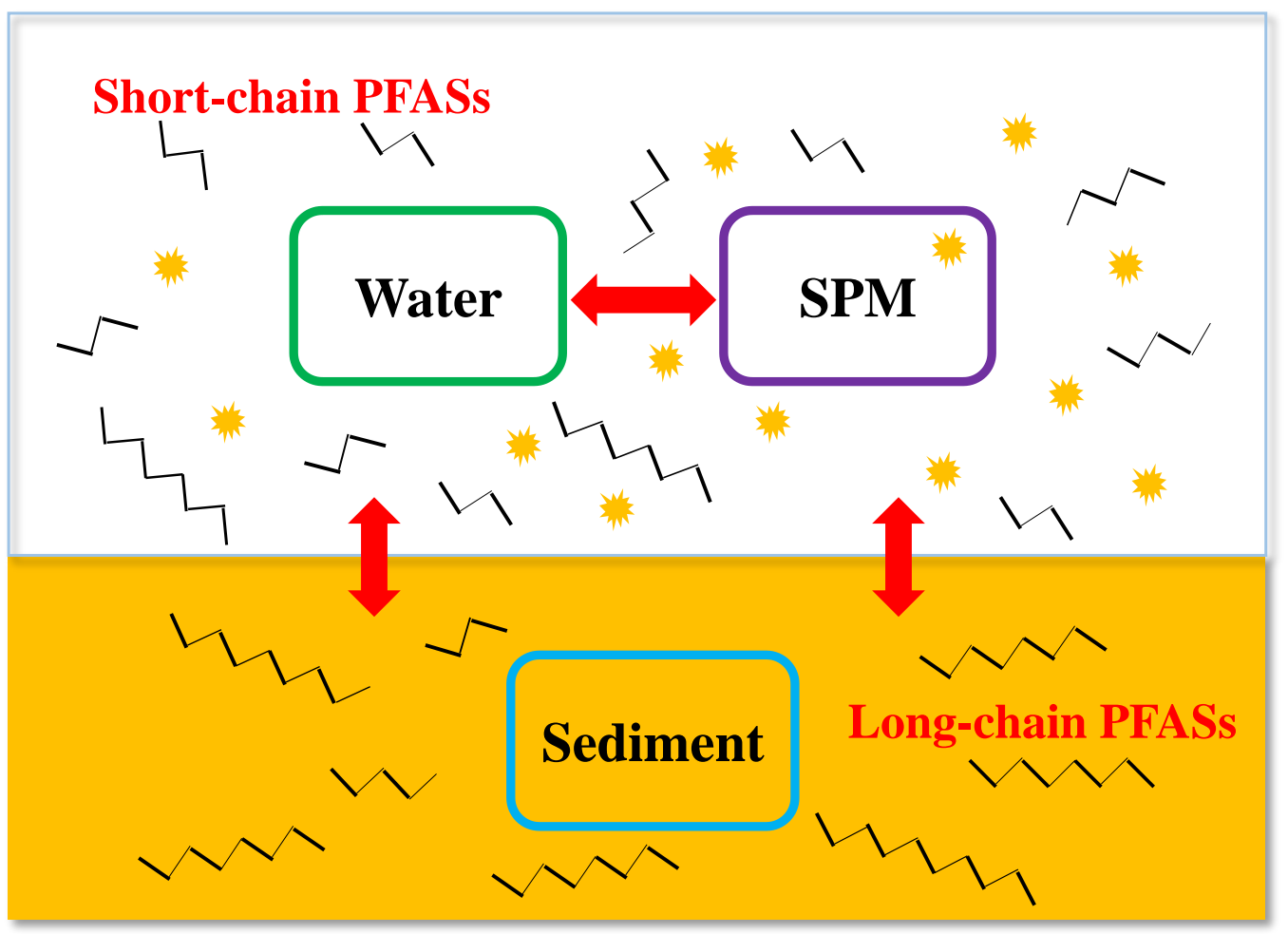

